analecta polit. | Vol. 9 | No. 16 | PP. 1-8 | enero-junio | 2019 | ISSN-e: 2390-0067 (en línea) |

Medellín-Colombia

doi: http://dx.doi.org/10.18566/apolit.v9n16.a01.

Cómo citar

este artículo en APA:

Alcántara, M. (2019). El

proceloso significado de lo político en el siglo xxI. Analecta Política, 9(16), $1-8$.

Fecha de recepción: 10.09.2018

Fecha de aceptación: 03.02.2019

\section{Editorial \\ El proceloso significado de lo político en el siglo XXI}

\section{MANUEL ALCÁNTARA SÁEZ}

Catedrático de la Universidad de Salamanca

Profesor visitante en la Universidad Pontificia Bolivariana,

Medellín-Colombia

Correo electrónico: malcanta@usal.es orcid.org/0000-0001-6483-3165 
La precisión conceptual es una de las finalidades primordiales en el ámbito académico. Este propósito es aún más perentorio si lo que se trata de definir es el propio término que denomina a una disciplina. Un objetivo teóricamente necesario e imprescindible a la hora de su operacionalización. La reflexión especulativa en torno al término "político" es uno de los grandes retos epistemológicos presente a lo largo de la evolución de la humanidad. Desde que hay constancia histórica no hay momento en que no se haya dejado de abordar el asunto integrando lo pensado en el contexto del momento y en consonancia con la evolución de diferentes paradigmas sobre los individuos y sus configuraciones tanto particulares como grupales.

La definición de lo político, de la política, ocupa un lugar central en la Ciencia Política una disciplina, como hoy se conoce, que sienta sus bases en los albores del siglo XX articulada sobre tres ejes fundamentales como son: el pensamiento liberal, la nueva sociedad de masas y el desarrollo de la universidad. El pensamiento liberal, en tanto que punta de lanza de estas ideas políticas, articula un tipo de régimen de equilibrio de poderes y de imperio del estado de derecho que apertura una dimensión nueva de lo político y de la política. Asuntos como la separación de poderes, el papel del estado y los propios fundamentos de la política con relación a su legitimidad legal-racional son sustanciales. La nueva sociedad surgida de la revolución industrial incorpora a millones de ciudadanos al juego sobre el usufructo y límites del poder definido por las reglas liberales; así mismo presiona sobre las viejas estructuras estatales en demandas de políticas públicas que satisficieran necesidades insoslayables. Finalmente, la universidad deja de ser el centro de estudio elitista que ha sido en los siglos anteriores para abrirse a las clases sociales emergentes e impulsar nuevos estudios con un perfil de mayor especialización sobre la base de viejos asuntos; no hay duda de que la expansión de la disciplina se va a dar en el seno de un proceso de profundo cambio social, de instituciones políticas novedosas y de enormes expectativas generales de movilización social gracias al acceso al conocimiento procesado, además, de forma diferente.

En el mundo anglosajón, donde este proceso se dio con un ritmo más acelerado, se pudo diferenciar perfectamente entre tres términos, politics, policy y polity, cuyas connotaciones en torno a las ideas de proceso, práctica y marco siempre tuvieron una notable dificultad para ser transferidas al español donde siempre ha estado presente la confusión terminológica. Sin llevar a cabo un repaso de cómo a través del siglo XX se vino configurando el escenario de discusión de lo político -la política- hay que destacar, entre otras, la tensión originada por las dos visiones contrapuestas entre Hannah Arendt y Carl Schmitt y el quiebre intermedio registrado por la propuesta de David Easton, así como las modificaciones que trajo consigo el neoinstitucionalismo. 
Para Arendt, prototipo de pensadora que reivindica afanosamente como modelo un mundo virtuoso, ser político equivale a ser una persona pública concernida por los asuntos públicos; además, la vida política de la ciudadanía constituye una opción moral libremente hecha por personas que podrían fácilmente haber escogido otra, siendo la asociación política una comunidad moral basada en la confianza, la amistad y el respeto mutuo, aspectos éstos sin los cuales el mundo político no existe. Frente a ella, el pensamiento de Carl Schmitt se alza como antagonista ya que para él lo político se basa en la distinción amigo-enemigo, una propuesta de calado radicalmente diferente. La revolución conductista, junto con la apropiación del concepto de "sistema" según su uso de Talcott Parsons, lleva a David Easton a su propuesta innovadora en la que lo político -la política- deja de ser un substantivo para quedar relegado a un puro adjetivo (sistema político). Lo que vale, por encima de todo, son relaciones, funciones, entre actores e instituciones, con flujos de demandas y de apoyos en búsqueda de equilibrios que resultan complicados. El poder es una mera recolocación de valores realizada por una autoridad legitimada (Easton). Después, los neoinstitucionalistas no hicieron sino apuntalar el edificio así construido dando un sentido diferente a las instituciones como constricciones que regulaban la interacción humana para evitar la incertidumbre (Douglas North).

Este es el estado de las cosas en la disciplina cuando se avizora el fin del siglo $\mathrm{XX}$, pero el escenario mundial cambia radicalmente en apenas una década como consecuencia de procesos que vienen incubándose. La caída del muro de Berlín acelera el proceso de globalización sobre la base de una profunda presencia del capitalismo transnacional y el desarrollo de las nuevas tecnologías de la información y de la comunicación aúpan más aun su avance. El escenario que ve el ascenso de nuevos jugadores en el panorama internacional, algunos en clave nacional (China, India, Rusia) y otros como conglomerados empresariales de insólito vigor insertos en la nueva economía de la materia oscura, de lo intangible y de lo simbólico ${ }^{1}$ (Apple, Alphabet-Google, Microsoft, Amazon, Facebook) trae consigo una cambio de enorme trascendencia sobre el papel de los estados nacionales que hasta entonces han constituido la unidad por excelencia donde se escenifica lo político -la política-. Pero el cambio más trascendental, acaece en la arena tecnológica al verse afectados dos niveles que hasta ese momento no han tenido impacto alguno: el significado de los nuevos soportes en manos de la gente y la capacidad de almacenar los datos generados y, posteriormente, interpretarlos por otros agentes.

1 Ver Haskel y Westlake (2018). 
Desde la perspectiva de la política los nuevos soportes en las tecnologías de la información y de la comunicación tienen un impacto enorme. Estas tecnologías conllevan cinco características independientes que configuran su frescura y su trascendencia. En primer lugar, son universales: a comienzos de 2019, y con apenas un cuarto de siglo de desarrollo, internet llega a 4.388 millones de personas, es decir, algo más de la mitad del planeta siendo la tasa de penetración en 17 países superior al 90\% (Galeano, 2019, 31 de enero). Conviene recordar que el teléfono fijo tardó 75 años para que su número de usuarios alcanzara a 100 millones de personas. En segundo lugar, son inmediatas, es decir, permiten la conectividad instantánea, en tiempo real. En tercer lugar, son portables ya que el uso de teléfonos celulares es potestad de dos tercios de la humanidad (el 52\% de la población mundial accede a internet por medio de su celular). En cuarto lugar, son reflexivas permitiendo la respuesta y la interconexión. Finalmente, facilitan la hiperconectividad por la que se puede estar a la vez en diferentes escenarios y al ser multifuncionales se posibilita al mismo tiempo, el uso de la voz, el empleo de cámara de fotos, relojes, agenda personal, quioscos de prensa e instrumentos de pago en las cada vez más habituales operaciones de comercio electrónico. Todo ello comporta aspectos nuevos de la interacción social como son la posibilidad de actuar anónimamente, la viralidad que un mensaje puede tomar en la red llegando a millones de usuarios en un tiempo muy reducido, el sentimiento de empoderamiento que logra llegar a sentir quien está en posesión de una terminal y la conciencia de pertenencia a una comunidad virtual.

Sin embargo, la aparente facilidad en la comunicación, que dinamizan las técnicas de propaganda, y en la movilización de simpatizantes de manera virtual -consiguiendo la aquiescencia explícita de los mensajes así como, en el mejor de los casos, hacer que concurran a la plaza-, tiene un lado menos positivo, Como ponen de relieve tanto Byung Chul Han $(2012,2014)$ como Franco Berardi (Massot, 2019, 20 de febrero) las tecnologías digitales están generando una mutación del ser humano y aceleran de forma tan vertiginosa el tiempo que no deja espacio para la pausa, la escucha o la capacidad crítica ponderada. Según Berardi, "los dispositivos tecnológicos se han convertido en una prótesis de nuestros cuerpos y en una herramienta de relación permanente con el mundo, devaluando así nuestra experiencia directa e inmediata de la realidad, afectando a las emociones, el psiquismo, la percepción y la relación con el otro".

La naturaleza de las nuevas tecnologías, por otra parte, dificulta la argumentación, sintetiza hasta tal extremo la información que hace muy difícil su comprensión contextual y facilita el incremento del impacto y de la velocidad de propagación de las noticias falsas (que siempre existieron) basadas en la emoción 
y en la segmentación de los ciudadanos en comunidades que actúan como cajas de resonancia. En este sentido, Nassim Taleb (2016, 14 de agosto) subraya cómo la mayoría falsifica públicamente sus preferencias para encajar dentro de su grupo adoptando posiciones extremas bajo la creencia equivocada de que son posiciones de consenso en el colectivo en que se quiere permanecer, cuando en el fondo lo son solo de sus cabecillas más gritones.

Berardi es muy pesimista con respecto a la democracia que concibe como "la dimensión donde nadie tiene razón porque todos tienen derecho a razonar conflictivamente en una sociedad abierta, porque no hay verdad, pues la verdad es el diálogo, y eso no significa nada hoy". Es la aceleración vivida en el universo de las tecnologías de la comunicación y de la información la que hace de manera absolutamente innovadora que el diálogo se verifique "entre el individuo y la pantalla, el individuo y la máquina, [habiendo] que respetar las reglas ineludibles de la máquina digital, que son las reglas de las finanzas. Ingresar en el mundo de la economía financiera significa entrar en una dimensión en la que las reglas están escritas en la máquina, y no se pueden discutir". Si la democracia es la posibilidad de discutir todo, principalmente las reglas, entonces, afirma, "la democracia está muerta", añadiendo: "la prueba la hemos visto en Grecia, en todos los lugares. Con la democracia no se puede cambiar nada. La revuelta de los chalecos amarillos es la última demostración". La desesperanza se extiende iluminado una democracia de audiencias, ya no tanto en el sentido de Charles Manin, porque ahora se configuran escenarios de manipulación de masas en red donde las emociones desempeñan un papel inusual. Aquí, la política pierde pie.

Pero hay un segundo ámbito generado por las nuevas tecnologías en una dimensión diferente y que se articula sobre la capacidad de capturar y de almacenar los datos generados, bien por los soportes utilizados en la comunicación o en el acceso a la información, así como mediante otras aplicaciones en lo que se ha venido a denominar el internet de las cosas, y, posteriormente, la capacidad de interpretar los datos y de volverlos operativos para posibilitar otras actuaciones por otros agentes. La nueva dimensión que vierte el mundo del big data y las posibilidades que ofrece la inteligencia artificial $^{2}$ constituyen un reto doble para la política.

2 "Lo que caracteriza a la naturaleza de la inteligencia artificial que hoy está en expansión no es la capacidad de duplicar nuestros recursos imaginativos, creativos o lúdicos para buscar finalmente superarlos, sino la aptitud para sobrepasar sin medida conocida el poder cerebral y cognitivo humano en ciertas tareas específicas, en vistas a garantizar la gestión de actividades existentes o nuevas de modo infinitamente más rápido, optimizado y fiable" (Sadin, 2019). 
Así, por una parte, la política, tras ser durante décadas la cenicienta de las ciencias sociales, en afortunada expresión de Harold Laski, por su inveterada carencia de datos y su excesiva dependencia de lo especulativo, se convierte en un receptáculo donde millones de datos esperan una interpretación. Cierto que recolectar más datos no garantiza que sean precisos, ni que estén actualizados y sean relevantes para cumplir nuestros objetivos, ni mucho menos que esos datos puedan ponerse per se al servicio de la justicia, la democracia, la igualdad y el bienestar, pero el cambio es irreversible.

Por otra parte, tampoco la inteligencia artificial elimina el problema de su propia gobernanza por la que los incentivos de las instituciones puedan estar alineados con los intereses de la población. Además, los avances en la inteligencia artificial llegan a cuestionar, por ejemplo, la existencia del libre albedrío como ha planteado recientemente de manera provocativa Yuval Noah Harari dibujando un panorama de cerebros que pueden ser pirateados ${ }^{3}$. La capacidad de desentrañar pautas de comportamiento, declaraciones de posiciones políticas, así como la propia gestación de redes posibilita interpretar tanto las múltiples interconexiones que llevan a cabo los individuos como el significado de algo tan profundo como son las emociones. La inteligencia artificial cuenta con tres facultades que la dotan de un notable vigor: poder interpretar situaciones de todo tipo, pudiendo evaluar de modo automatizado estados de hecho en el seno de un corpus de datos, detentar el poder de sugerir diferentes soluciones para abordar un mismo problema, y ser capaz de manifestar autonomía decisional, es decir, tiene la capacidad de emprender acciones sin validación humana previa (Sadin 2019).

Si durante mucho tiempo la política ha descansado en cuestiones tales como la construcción de la confianza o la definición de la identidad sobre las que los estudiosos conocían muy poco acerca de sus mecanismos constitutivos ${ }^{4}$, ahora se abre una oportunidad única a la hora de navegar en el proceloso mundo del yo. El propio Yuval Noah Harari subraya que "el liberalismo ha desarrollado un impresionante arsenal de argumentos e instituciones para defender las libertades

3 El autor israelí señala que "para conseguir piratear a los seres humanos, hacen falta tres cosas: sólidos conocimientos de biología, muchos datos y una gran capacidad informática. La Inquisición y el KGB nunca lograron penetrar en los seres humanos porque carecían de esos conocimientos de biología, de ese arsenal de datos y esa capacidad informática. Ahora, en cambio, es posible que tanto las empresas como los Gobiernos cuenten pronto con todo ello y, cuando logren piratearnos, no solo podrán predecir nuestras decisiones, sino también manipular nuestros sentimientos" (Harari, 2019, 5 de enero).

4 Aunque su abordaje teórico haya sido proceloso. Ver al respecto Fukuyama (1998 y 2019). 
individuales contra ataques externos de Gobiernos represores y religiones intolerantes, pero no está preparado para una situación en la que la libertad individual se socava desde dentro y en la que, de hecho, los conceptos libertad e individual ya no tienen mucho sentido" (Harari, 2019, 5 de enero). De esta suerte, una vez desdibujado el terreno de lo público, se abren nuevos terrenos de análisis donde las ciencias de la computación, las neurociencias, la bioingeniería y la psicología tienen mucho que decir, así como todo el entramado de conocimientos en torno al mundo de la comunicación.

Lo político se desvanece, además, en el entramado de las relaciones que impulsan las nuevas tramas de la economía. El neoliberalismo triunfa, no solo por sus propuestas económicas en clave del paroxismo del consumismo y del triunfo de la faceta financiera de la economía, sino por haber incorporado a la sociedad sólidas pautas culturales. La idea de competencia irrestricta entre sus miembros, de soledad profunda y de individualismo egoísta a ultranza guía el comportamiento de las personas. En este sentido es interesante constatar cómo las expresiones contestatarias que se dan paulatinamente son configuradas como anti políticas. La sociedad líquida, en términos de Zygmund Bauman ${ }^{5}$, se ve acompañada en el actual momento, ya avanzado el siglo XXI, por instituciones sólidas que punteaban nuestra realidad que, sin embargo, se desvanecen. Las transformaciones en el universo de los partidos políticos son una clara evidencia en la medida de la pérdida masiva de confianza en ellos, la centralidad de los candidatos sobre las maquinarias partidistas y la pérdida de identificación del electorado con sus etiquetas. Además, se ha dado paso a una realidad marcada por la precariedad, el ritmo cambiante e inestable, la celeridad de los acontecimientos y la dinámica agotadora y con tendencia al referido individualismo de las personas.

Todo ello se vincula con una política que requiere de una definición innovadora que, hoy por hoy, se resiste a aparecer. Es indudable, no obstante, que el camino emprendido por Susskind (2018) es la vía a seguir. Su propuesta se articula, precisamente, bajo la premisa de que la forma en que se gestione la información (cómo se obtenga, se almacene, se analice y se comunique) está estrechamente ligado a la organización de la vida colectiva y en común que es la esencia de la política. La gobernanza de la una es la de la otra. Para Susskind en la nueva era digital, donde la tecnología desempeña el papel central, serán cada vez más poderosos quienes controlen las tecnologías que posibilitan los tres vectores del poder

5 Ver su libro Modernidad líquida. México: Fondo de Cultura Económica. 
que tomarán tres formas: fuerza, escrutinio y control de la percepción. Los que queden fuera de este escenario cada vez serán más vulnerables. Lo político tiene que ver con todo ello.

\section{Referencias}

Fukuyama, F. (1998). La confianza. Barcelona: Ediciones B.

Fukuyama, F. (2019). Identidad. Deusto.

Galeano, S. (2019, 31 de enero). El número de usuarios de Internet en el mundo crece un 9,1\% y alcanza los 4.388 millones. Marketing 4 Ecommerce. Recuperado de https://marketing4ecommerce.net/usuarios-internet-mundo/

Han, B.-Ch. (2012). La sociedad del cansancio. Barcelona: Herder.

----- (2014). En el enjambre. Barcelona: Herder.

Harari, Y. N. (2019, 20 de febrero). Los cerebros "hackeados" vota. El País. Recuperado de https://elpais.com/internacional/2019/01/04/actualidad/1546602935_606381.html

Haskel, J. \& Westlake, S. (2018). Capitalism without Capital. The Rise of the Intangible Economy. Princeton: Princeton University Press.

Massot, J. (2019, 20 de febrero). El problema es cómo la pantalla se ha apoderado del cerebro. El País. Recuperado de https://elpais.com/cultura/2019/02/18/actualidad/1550504419_263711.html

Sadin, É. (2019) La inteligencia artificial: el superyó del siglo XXI. Nueva Sociedad, enero-febrero 2019. http://nuso.org/articulo/la-inteligencia-artificial-el-superyo-delsiglo-xxi/?utm_source=email\&utm_medium=email

Susskind, J. (2018). Future Politics. Living Together in a World Transformed by Tech. Oxford: Oxford University Press.

Taleb, N. N. (2016, 14 de agosto). The most intolerant wins: the dictatorship of the small minority. Incerto. Recuperado de https://medium.com/incerto/the-most-intolerantwins-the-dictatorship-of-the-small-minority-3f1f83ce4e15 consultado el 21.02.19 